\title{
CEFALOGRAMA DE LA DEGLUCIÓN: Trazado, Valores Estándar y Desvíos
}

\author{
Norma Chiavaro6 \\ Presidente Fundador de la Academia Latinoamericana de Disfunciones Estomatognáticas (ALDE) \\ Autora del Método Chiavaro. Técnica NM-F. aplicable al diagnóstico y terapéutica de las Disfunciones \\ Estomatognáticas. \\ Directora y Dictante de Posgrados Universidad del Salvador(USAL)Prof. a cargo del Área Fonoestomatología \\ y Titular de las materias de MO de Carrera de Lic. en Fonoaudiología, Facultad de Medicina y Ciencias de \\ la salud USAL. \\ Prof. a cargo del área M.O. en Carrera de Especialidad en Ortodoncia y Ortopedia Maxilar del Círculo \\ Argentino de Odontología. \\ Directora del CEMEC Centro Método Chiavaro. Asistencia, Investigación y Docencia.
}

El objetivo de trabajo es presentar el Cefalograma de la Deglución a partir del trazado en cefalometría digital de puntos y planos que conforman el Triángulo de la Deglución, así como la evolución teórica del triángulo de la deglución como objeto de investigación.

Los avances tecnológicos y digitales en el transcurrir del tiempo nos permitió acceder mediante estudios objetivos a modificar conceptos y creencias apoyándonos en evidencia científica.

1. 1995-Aparecen las primeras experiencias de estudios objetivos "Electromiografía de superficie" al que tengo acceso mediante el Sistema Biopack, de uso en Odontología para el estudio de las Disfunciones Témporo-Mandibulares donde se demuestraba la intervención de los músculos Mandibulares como determinantes en la Deglución.

2. 2001- Chiavaro presentó a la comunidad científica la "Teoría de la etapa Mandibular de la Deglución. Concepto Neuromuscular-Funcional. Etapas de la deglución según la intervención de las Unidades Funcionales neuromusculares". Realizo divulgación en eventos científicos, clases y publicaciones. Más adelante lo publico en el Cap. 4 del libro "Tratamiento de la Deglución. Actuación de Fonoaudiólogos en Diferentes países". Organizadora Irene Queiroz Marchesan Editorial Pulso 2005

3. 2009- Chiavaro profundiza el particular de la participación neuromuscular en la deglución e inicia la observación del rol que entonces tenían las Articulaciones Occípito-Atlo-Axoidea y las ATM en el primer tiempo de la Deglución quienes por lo tanto serían responsables a partir de su fijación de las estabilidades Cráneo-Cervical y Cráneo-mandibular las cuales serían previas y necesarias para la estabilidad Linguo-palatal. El objetivo, de este trabajo fue determinar dichos puntos de fijación uniéndolos con líneas conectoras entre ellos lo cual nos daría idea de la línea de recorrido de la energía circulante. Surge así una trayectoria que va de atrás hacia arriba y adelante y luego de adelante hacia atrás nuevamente regresando al punto de inicio en la articulación occipito-atloidea quedando definida una circulación con forma triangular en cualquier plano que se lo considere.

6. Fonoaudióloga, Presidente Fundador de la Academia Latinoamericana de Disfunciones Estomatognáticas (ALDE). Autora del Método Chiavaro. Técnica NM-F. aplicable al diagnóstico y terapéutica de las Disfuncio nes Estomatognáticas. Directora y Dictante de Posgrados Universidad del Salvador (USAL) Prof. a cargo del Área Fonoestomatología y Titular de las materias de MO de Carrera de Lic. en Fonoaudiología, Facultad de Medicina y Ciencias de la salud USAL. Prof. a cargo del área M.O. en Carrera de Especialidad en Ortodoncia y Ortopedia Maxilar del Círculo Argentino de Odontología. Directora del CEMEC Centro Método Chiavaro. Asistencia, Investigación y Docencia. 
A partir de este trazado se descubre que la energía circulante es su participación del impulso hacia arriba y atrás del velo de paladar cuando se eleva hacia la pared posterior faríngea produciéndose el cierre del esfínter velo-faríngeo (2da etapa de la deglución). Dicho acoplamiento y sellamiento del velo sobre la pared posterior faríngea se encuentra exactamente sobre la línea de base del mismo triangulo en donde la misma energía en su trayectoria de regreso hacia el punto de origen del recorrido promueve que impacte sobre la superficie cóncava de la pared posterior faríngea. Allí estaría la cuarta estabilidad determinada por dicho acoplamiento absolutamente necesario en su efecto valvular ya que de esta acción valvular depende la siguiente, la válvula laríngea.

Se presenta entonces a la comunidad científica el texto: "Estabilidades requeridas en la Deglución. Trazado del Triángulo de Estabilidad de la Deglución" Divulgación científica en numerosos eventos, clases y publicaciones a partir de 2005.

1. 2011- Chiavaro publica: "Deglución. Estabilidades y eventos neuromusculares" Cap. 3 del libro de "Funciones y Disfunciones Estomatognáticas" Editorial Akadia. Buenos Aires 2011.

2. 2013- "Deglución: Teoría del Tiempo mandibular. Eventos neuromusculares y procesos de estabilidad que participan de la Deglución" Cap. 10 del libro "Motricidad Orofacial. Fundamentos basados en evidencias." Coordinadores: Franklin Susanibar, David Parra, Alejandro Dioses. Editorial Eos 2013.

3. 2014- Luego de varios años de estudios y mediciones la Dra. Graciela Porta y luego el Dr. Carlos Ausbruch colaboran en la organización de un Cefalograma de la deglución con el trazado y la medición del Triángulo de estabilidad para ser aplicado a Cefalometría y lo denominan "Cefalograma Chiavaro".

Dicho registro estadístico de datos tiene como base de sustentación la necesidad de generar parámetros de normalidad y desvíos a partir de trazados de puntos cefalométricos determinando medidas lineales y angulares correspondientes a los distintos biotipos Iniciando por el hallazgo de medida standard en biotipo Mesofacial. Relación con desvíos esqueletales y oclusales en los distintos biotipos. Surge el programa de medición Digitalcef aplicable a la cefalometría digital y con el cual se realizan los estudios estadísticos.

El objetivo de esta propuesta es la objetividad en el estudio de la deglución para continuar a partir de un estudio objetivo como el Cefalograma constatar posibilidades y resultados terapéuticos de manera objetiva. Esto será una herramienta apropiada para el diagnóstico y programación terapéutica del Odontólogo ortopedista, ortodoncista, cirujano máxilo-facial y del Fonoaudiólogo. Este estudio posibilita ampliamente la relación Fonoaudiología-Odontología ya que participan ambos del estudio diagnóstico. 